\title{
7. Geomagnetic pc4 Pulsations Associated with a Storm Sudden Commencement caused by a Cavity Resonance
}

\author{
By Yoshio Kato, M. J. A., and Yutaka Tonegawa*) \\ (Communicated March 12, 1991)
}

\begin{abstract}
A quasi-sinusoidal geomagnetic pulsation having a period of about 100-s is called pc4 and it is also associated with a storm sudden commencement. Concerning the problem of exciting mechanism, the authors compared the magnetic records obtained from the DE-1 spacecraft and the ground-based induction magnetograms obtained at Kanoya. Our conclusion is that the pulsations with 100 -s period associated with ssc of magnetic storm are caused by global cavity resonance oscillations.
\end{abstract}

Key words: pe4 pulsation; storm sudden commencement; cavity resonance.

1. Introduction. It is well known that geomagnetic pe4 pulsations of quasisinusoidal damped oscillations of 100 -s period are associated with storm sudden commencement. These pulsations are attracted by many investigators, who have discussed on the exciting mechanism of the pulsations.

The geomagnetic storm that occured on March 13, 1989, is displayed with a magnetogram obtained at Kakioka in Fig. 1. Fig. 2 shows one of the examples of the pc4 pulsations recorded during the ssc on the induction magnetogram at Kanoya (latitude $31^{\circ} 25^{\prime} .2$, longitude $130^{\circ} 52^{\prime} .6$, geomagnetic latitude $21^{\circ} .1$ geomagnetic longitude $199^{\circ} .8$ ).

Recently Cahill, et al. [1990] observed with a fluxgate magnetometer onboard Dynamic Explorer 1 (DE-1) a strong east-west component of magnetic oscillations with

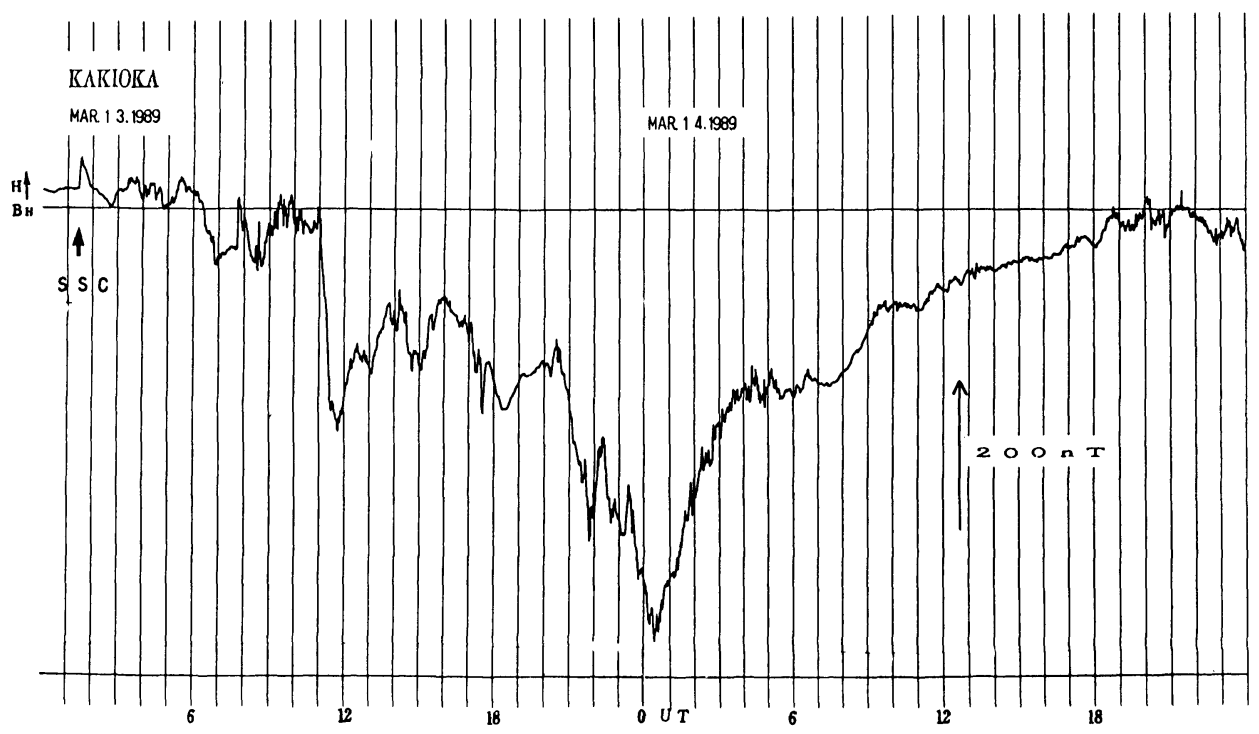

Fig. 1. Magnetic record of the storm observed at Kakioka on March 13, 1989.

*) Department of Aeronautics and Astronautics, Tokai University, Hiratsuka, Kanagawa, Japan. 


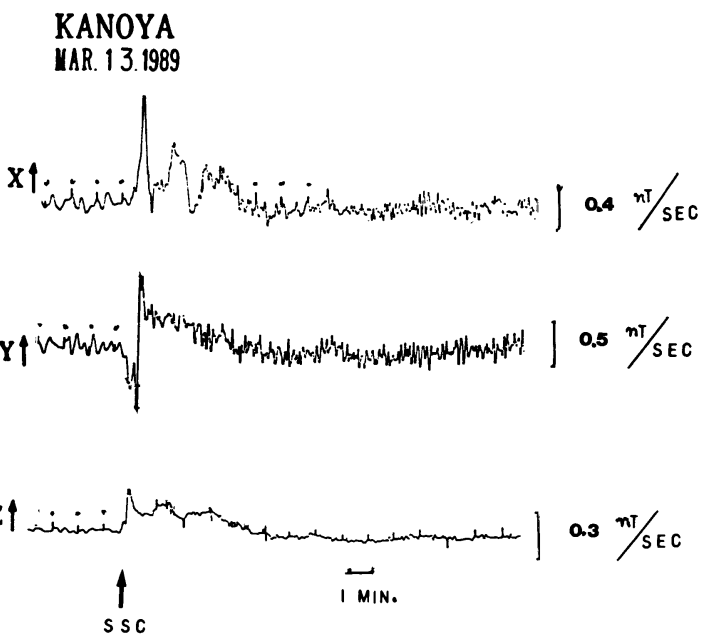

Fig. 2. Geomagnetic pc4 pulsation associated with ssc observed at Kanoya on March 13, 1989.

100-s period being excited by a storm sudden commencement of July 13, 1982. They suggested that the oscillations are caused by a cavity resonance.

In the present paper, we compare the satellite-based magnetic pulsation with a ground-based pulsation recorded at Kanoya, Japan, during the same storm sudden commencement of July 13, 1982. It is found that the period and the wave pattern of the ground-based pulsation are similar to those observed in space by DE-1 in spite of the quite different locations between the two observing sites. Therefore, we may offer a new evidence that the pc4 pulsations associated with a storm sudden commencement are caused by the global cavity resonance oscillations.

2. Geomagnetic pe4 pulsations simultaneously observed in space and on the ground. A toroidal standing wave excited by ssc of July 13, 1982, as detected by DE-1 has been investigated by Cahill et al. (1990). The satellite was located near the plasmapause of $\mathrm{L}=4.5$ during the event. A strong east-west component of the oscillations having the period of 100 -s was clearly recorded. There were also two compressional components of the 100 -s and 300 -s oscillations. They have suggested that the compressional oscillations excited the cavity resonances and then coupled to the toroidal standing waves at the satellite location.

On the other hand, pc4 pulsations observed at Kanoya $(\mathrm{L}=1.2)$ during this sse of July 13, 1982, are shown in Fig. 5. A damped-type oscillation was clearly detected in the east-west $(\mathrm{Y})$ component, although the north-south $(\mathrm{X})$ component was not available because of a scaling-out. The magnetic storm of July 13, 1982, is displayed with the magnetogram obtained at Kakioka, in Fig. 4.

Fig. 3 shows a schematic model of the magnetosphere with the position of DE-1 by the letter D, and Kanoya by the letter K. During the wave event, DE-1 and Kanoya were located at $\sim 1530$ MLT and $\sim 0130$ MLT, respectively.

Fig. 6 shows the pc4 pulsation of the east-west component which is transcribed from the record at Kanoya in Fig. 5.

Fig. 7 shows the frequency spectrum of this pc4 event with the spectral peak at about $9 \mathrm{mHz}$. It is interesting that the waveform and the period of this event is quite similar to the east-west oscillation observed at the DE-1 spacecraft. 


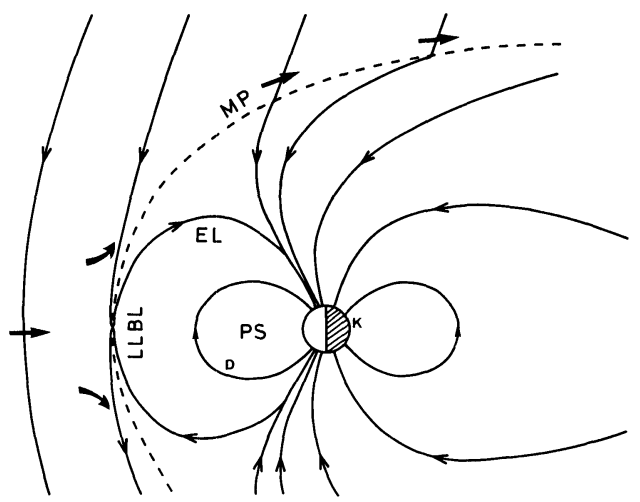

Fig. 3. Schematic model of the magnetosphere showing the position of DE-1 (D) and Kanoya (K).

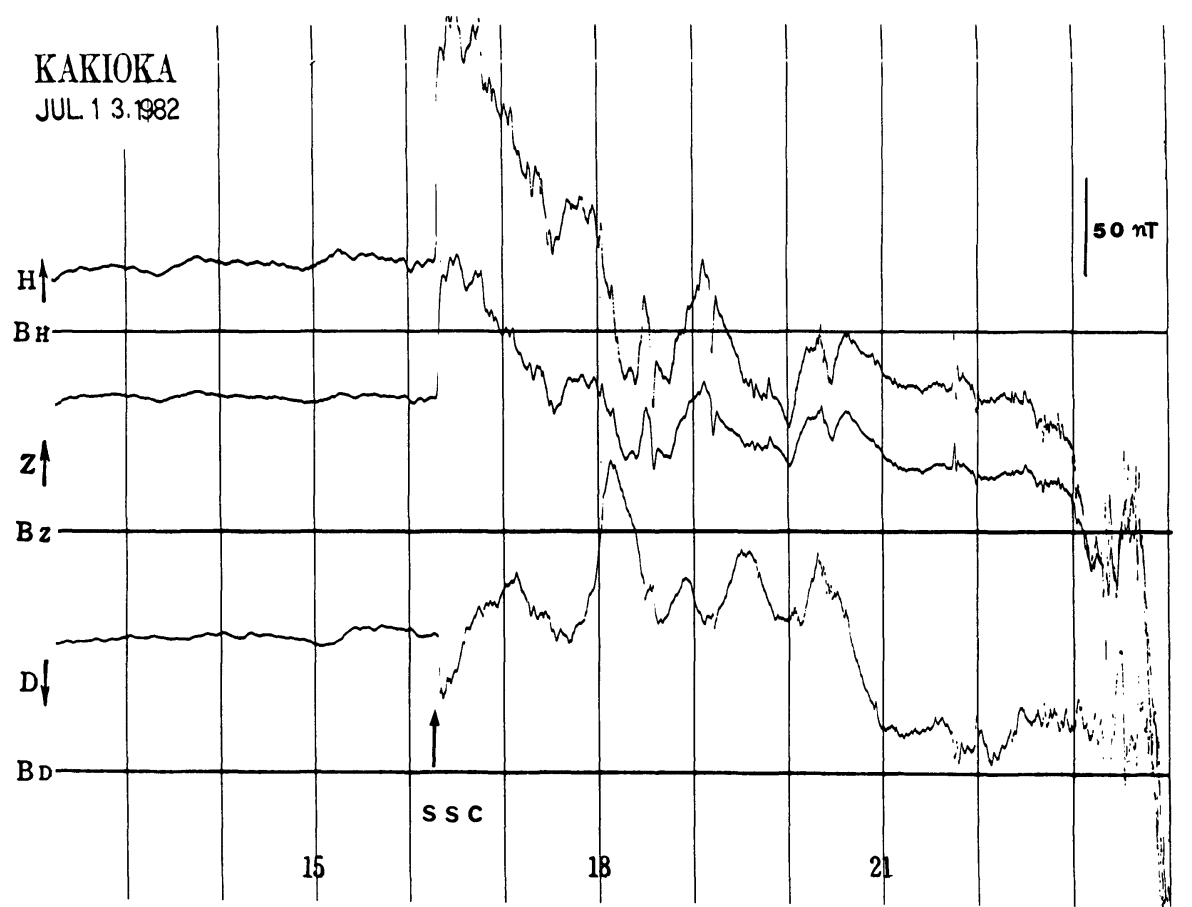

Fig. 4. Magnetic storm observed at Kakioka on July 13, 1982.

3. Conclusion and discussion. Our conclusion will be summarized as follows:

(1) Some compressional waves are excited at the boundary of the magnetosphere at the initial time of storm sudden commencement.

(2) The waves propagate into the magnetosphere.

(3) These waves couple with toroidal standing waves at a certain $\mathrm{L}$ shell, for example, at the plasmapause.

The comparison between the pc4 pulsations observed at DE-1 and Kanoya derived an 
KANOYA

JUL. 13.1982

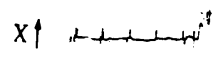

$0.4 n t / s E C$
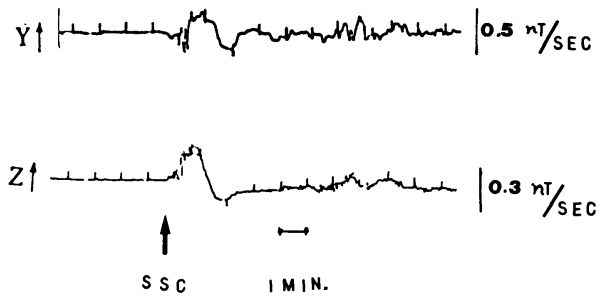

Fig. 5. Geomagnetic pc4 pulsation associated with ssc observed at Kanoya on July 13, 1982. The oscillation in the NS component is masked by the followed oscillations.

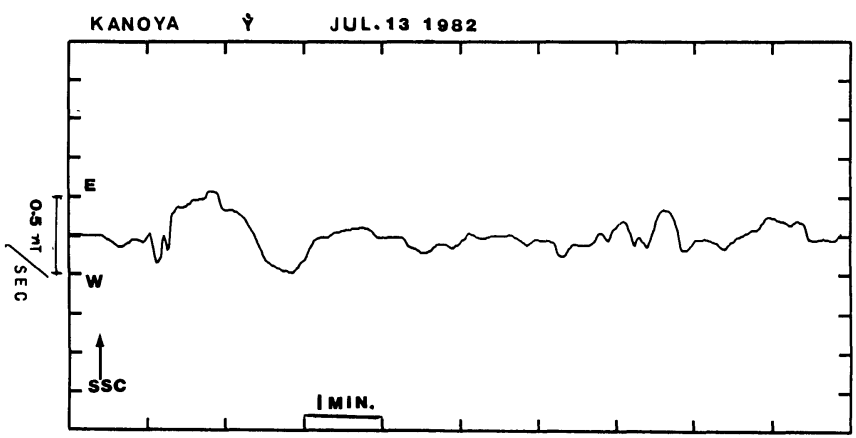

Fig. 6. Geomagnetic pc4 pulsation associated with ssc in the east-west component as transcribed from Fig. 5 .

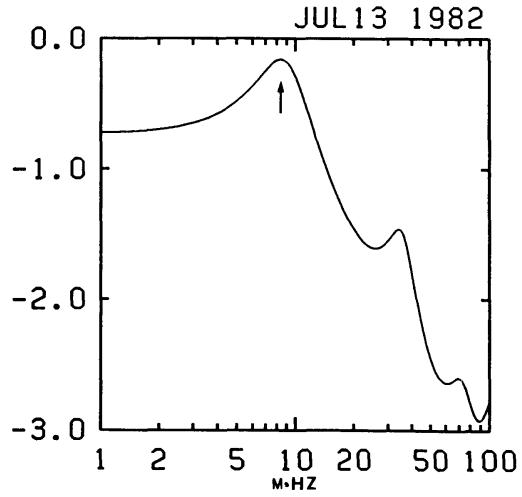

Fig. 7. Frequency spectrum of the east-west component of the pc4 pulsation associated with ssc of the magnetic storm of July 13, 1982. 
amazing result that both oscillation periods at quite different locations were well coincident with each other. The magnetic pulsation observed by $\mathrm{DE}-1$ at $\mathrm{L}=4.5$ in the dayside magnetosphere indicated the dominant oscillation in the east-west component with 100-s period. The compressional components with the periods of 100 -s and 300 -s were also observed simultaneously. It has been suggested by Cahill et al [1990] that the ssc gave rise to the compressional eigenmodes with the 100 -s and 300 -s periods as the magnetosphere cavity resonances, and that the 100-s compressional eigenmode coupled to the toroidal standing wave of the fundamental mode at $\mathrm{L}=4.5$.

On the other hand, the ground-based observation at Kanoya in the night hemispher $(\mathrm{L}=1.2)$ shows a 100 -s pulsation clearly in the east-west component in this case. The magnetic oscillation in the east-west component on the ground corresponds generally to the magnetic oscillation in the north-south component in the magnetosphere because of the $90^{\circ}$ rotation of the principal axis through the ionosphere. Since we have no available data in the north-south component at Kanoya, we cannot discuss about the toroidal waves at $\mathrm{L}=1.2$ from the present data.

It is therefore suggested that the cavity resonance oscillation excited by the ssc directly penetrated to the low-latitude night side ionosphere in this case and was detected on the ground as the pc4 pulsation with the 100-s period.

It must be noted that the pc4 pulsations appear within only the first few minutes of the initial increasing stage at the low-latitude ground station $(\mathrm{L}=1.2)$. The limited appearance on the ground may suggest that the resonant oscillation occurs widely in the whole cavity only in the initial stage.

Acknowledgment. The authors express sincere thanks to the Director of the Kakioka Magnetic Obsevatory for his kind offering of the copies of the magnetic records.

\section{Reference}

L. J. Cahill. JR. et al.: Toroidal standing waves excited by a storm sudden commencement; DE-1 observations, J. G. R., vol. 95, no. A6, pp. 7857-7867 (1990). 